\title{
Gastric Adenocarcinoma of the Fundic Gland Type: A Clinicopathological Observation
}

\author{
Lixin Wang, Gang Chen and Jingui Jiang \\ Department of Pathology, Jinhu County People's Hospital, Huaian, Jiangsu Province, China
}

\begin{abstract}
We investigated the clinicopathological features, diagnosis and differential diagnosis of a patient with gastric adenocarcinoma of the fundic gland type (GA-FG). Endoscopic examination, histomorphological and pathological features were assessed in a 38year female patient with GA-FG, who presented with upper abdominal pain. Upon endoscopic examination, we observed a smashed area around the cardia. Biopsy and pathological examinations revealed tumor tissue similar to fundic glands composed of parietal and chief cells. Endoscopic submucosal dissection was then performed and the tumor was found to infiltrate into the submucosa.

GA-FG is a new tissue type of gastric cancer with unique clinicopathological features, with a good prognosis, and no tendency to relapse.
\end{abstract}

Key Words: Gastric adenocarcinoma, Fundic gland type, Endoscopy.

How to cite this article: Wang L, Chen G, Jiang J. Gastric Adenocarcinoma of the Fundic Gland Type: A Clinicopathological Observation. J Coll Physicians Surg Pak 2021; 31(10):1237-1238.

\section{INTRODUCTION}

Gastric adenocarcinoma of the fundic gland type (GA-FG) originates from the deep regions of the non-atrophic normal gastric fundic glands. It is a new type of adenocarcinoma observed in gastric cancers. This type of tumor was first reported in Japan in $2020 .{ }^{1}$ It is defined as a tumor with differentiation into the gastric fundic type glands and is positive for pepsinogen I on immunohistochemistry (IHC). ${ }^{2} \mathrm{GA}-\mathrm{FG}$ s are rare and account for $1.6 \%$ of all gastric carcinomas. ${ }^{3}$ It is difficult to diagnose and it can be misdiagnosed due to its rarity and lack of insight into its morphological characteristics. This article retrospectively analyses a patient with GA-FG with a review of relevant literature to assess its clinic pathological profile.

\section{CASE REPORT}

A 38-year female presented to our hospital with the complains of upperabdominal pain. Endoscopy of the upper digestive tract revealed gastric mucosa to be smooth with no ulcer or erosion in most of the stomach. However, there was an erosion present, about $0.5 \mathrm{~cm}$ in diameter, in the region of the cardia. Biopsy was takenforhistopathological examination thatGA-FGtype. Endoscopic submucosal dissection (ESD) was then performed.

Correspondence to: Dr. Jingui Jiang, Department of Pathology, Jinhu County People's Hospital, Huaian, Jiangsu Province, China

E-mail: 15189521184@163.com

Received: November 20, 2019; Revised: February 22, 2020;

Accepted: March 09, 2020

DOI: https://doi.org/10.29271/jcpsp.2021.10.1237
Microscopically, the surface of the lesion was eroded. The tumor itself was composed of irregular, complex glands, disorderly arranged and fused with each other. Both parietal and chief cells were present in the neoplastic glands. The number of chief cells was significantly higher as compared to acidophilic cells (Figure 1). The neoplastic glands were seen infiltrating into the muscularis mucosae and invading about $0.5 \mathrm{~mm}$ into the submucosa (Figure 2).

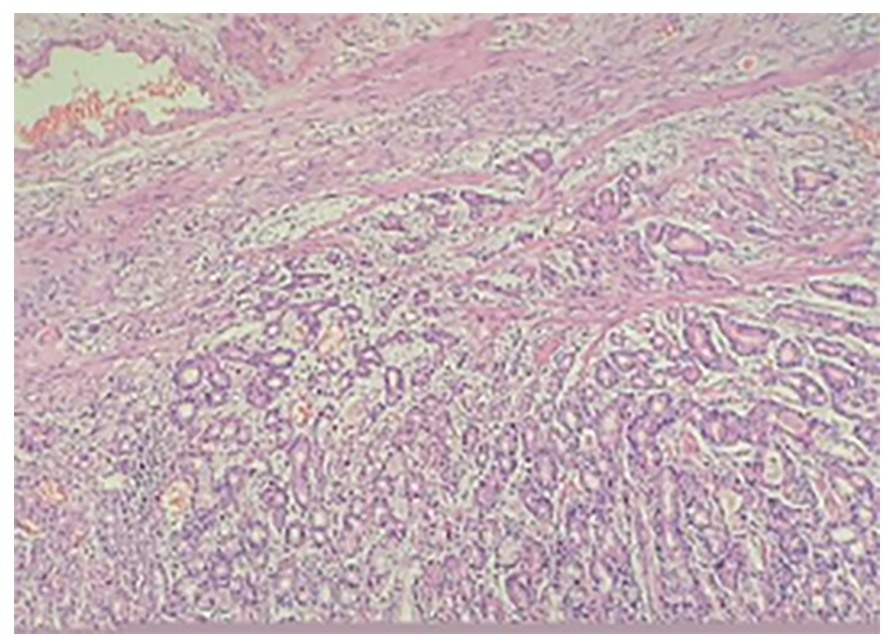

Figure 1: Low-power photomicrograph showing the eroded tumor surface. The tumor itself is composed of disorderly arranged, complex glands with infiltrating into the muscularis mucosae (H\&E, ×100).

Immunohistochemical (IHC) staining revealed tumor to be positive for pepsinogen I, focally positive for MUC 6 and weakly positive for H/K-ATPase. In addition, scattered positivity was observed for Ki-67, MUC 2, and p53. IHC staining for MUC 5AC was negative. 


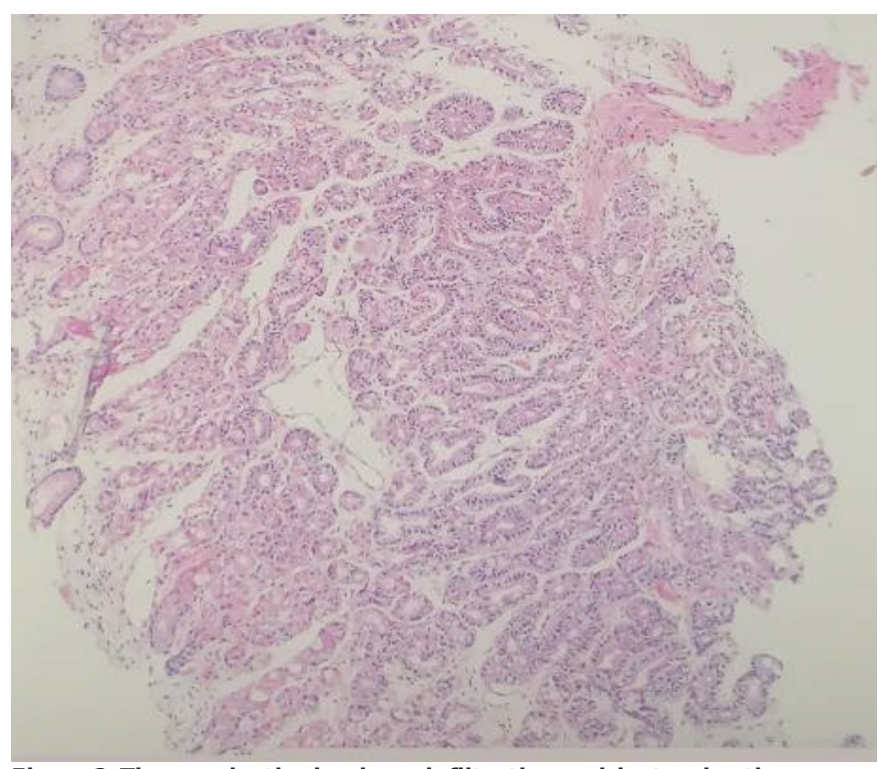

Figure 2: The neoplastic glands are infiltrating and destroying the muscularis mucosae $(H \& E, \times 100)$.

\section{DISCUSSION}

The pathogenesis of GA-FG is unclear. However, it may be associated with the use of proton pump inhibitors leading to gastric acid changes, ${ }^{4}$ or acute damage to the gastric fundus gland mucosa leading to the differentiation of primary cells into mucous cells. ${ }^{5}$ At present, the pathogenic mechanism of GA-FG is thought to be associated with CTNNB1 and AXIN gene mutations, and nuclear aggregation of $\beta$-catenin protein leading to the activation of the $W n t / \beta$-catenin pathway. In addition, GNAS mutations in a few patients have been associated with GA-FG pathogenesis. ${ }^{6} \mathrm{GA}-\mathrm{FG}$ lesions are usually small and have a good prognosis. Endoscopic treatment is recommended. GA-FG has been shown to infiltrate the submucosa to a depth of $0.5 \mathrm{~mm}$, or even up to $4 \mathrm{~mm}$. However, because there have been only a few GA-FG cases of deep infiltration into the mucosa, lymph node metastasis and survival rates are unknown. Whether the same standard treatment protocols that are used for common early gastric cancers could be administered for GA-FG is yet to be determined. Furthermore, whether additional surgery is required after ESD is still unclear. Methods to differentially diagnose GA-FG tumors from gastric fundus polyps, deep cystic gastritis, differentiated gastric cancers, and neuroendocrine tumors are required.
In summary, GA-FG is a new type of low-grade stomach cancer with unique clinical and pathological features. The prognosis for GA-FG is good with a low recurrence rate. ESD resection is the main treatment strategy; however, long-term follow-ups are required.

\section{PATIENT'S CONSENT:}

Informed consent was obtained from the patient.

\section{CONFLICT OF INTEREST:}

The authors declared no conflict of interest.

\section{AUTHORS' CONTRIBUTION:}

LW: Concept, data collection and interpretation, literature search and manuscript writing.

GC: Concept, data collection and interpretation.

JJ : Concept.

\section{REFERENCES}

1. Tsukamoto T, Yokoi T, Maruta S, Kitamura M, Yamamoto T, Ban $\mathrm{H}$, et al. Gastric adenocarcinoma with chief cell differentiation. Pathol INT 2007; 57(8):517-22. doi: 10. 1111/j.1440-1827.2007.02134.x.

2. Ueyama H, Yao T, Nakashima Y, Hirakawa K, Oshiro Y, Hirahashi M, et al. Gastric adenocarcinoma of fundic gland type (chief cell predominant type): Proposal for a new entity of gastric adenocarcinoma. Am J Surg Pathol 2010; 34(5):609-19. doi: 10.1097/PAS.0b013e3181d94d53.

3. Hidaka Y, Mitomi H, Saito T, Takahashi M, Lee SY, Matsumoto $\mathrm{K}$, et al. Alteration in the $\mathrm{Wnt} / \beta$-catenin signaling pathway in gastric neoplasias of fundic gland (chief cell predominant) type. Hum Pathol 2013, 44(11): 2438-48. doi: 10.1016/j.humpath.2013.06.002.

4. Chen O, Shao ZY, Qiu X, Zhang GP $\square$ Multiple gastric adenocarcinoma of fundic gland type: A case report. World J Clin Cases 2019; 7(18):2871-8. doi: 10.12998/wjcc. v7.i18.2871.

5. Imagawa A, Sano N. Gastric adenocarcinoma of the fundic gland (chief cell-predominant type) with brownish pigmentation J. Gastrointest Endosc 2018; 87(5):1358-9. doi: 10.1016/j.gie.2017.10.016.

6. Benedict MA, Lauwers GY, Jain D. Gastric adenocarcinoma of the fundic gland type: Update and literature review. Am J Clin Pathol 2018; 149(6):461-73. doi: 10.1093/ajcp/ aqy019. 